\title{
Long Non-Coding RNA MALATI as a Detection and Diagnostic Molecular Marker in Various Human Cancers: A Pooled Analysis Based on 3255 Subjects
}

This article was published in the following Dove Press journal: OncoTargets and Therapy

Yue Zhao ${ }^{1,2, *}$

Ya-qi Yu ${ }^{1, *}$

Song $\mathrm{You}^{3}$

Chang-mao Zhang ${ }^{4}$

Liang $\mathrm{Wu}^{5}$

Wenxiu Zhao'

Xiao-min Wang'

'Department of Hepatobiliary Surgery, Zhongshan Hospital, Xiamen University, Fujian Provincial Key Laboratory of Chronic Liver Disease and Hepatocellular Carcinoma, Xiamen, Fujian 361004, People's Republic of China; ${ }^{2}$ Department of General, Visceral, and Transplant Surgery, Ludwig-Maximilians-University Munich, Munich 81377, Germany; ${ }^{3}$ Mengchao Hepatobiliary Hospital of Fujian Medical University, The Liver Center of Fujian Province, Fujian Medical University, Fuzhou 350025, People's Republic of China; ${ }^{4}$ Faculty of Clinical Medicine, Fujian Medical University, Fuzhou, Fujian, People's Republic of China; ${ }^{5}$ Department of Nephrology, The First Affiliated Hospital of Xiamen University, Xiamen, Fujian 361003, People's Republic of China

*These authors contributed equally to this work

Correspondence: Xiao-min Wang Department of Hepatobiliary Surgery, Zhongshan Hospital, Xiamen University, Fujian Provincial Key Laboratory of Chronic Liver Disease and Hepatocellular Carcinoma, Xiamen, Fujian 361004, People's Republic of China Email wxm2203@xmu.edu.cn
Purpose: Accumulating studies have explored the potential diagnostic value of lncRNA MALAT1 in various cancers. However, there are still inconsistent results in diagnostic accuracy and reliability in individual studies. The aim of this pooled study was to summarize the overall diagnostic capacity of lncRNA MALAT1 in cancer detection and diagnosis.

Methods: Eligible studies satisfying the inclusion criteria were screened and selected from the online database. All statistical analyses were performed using Stata 14.0.

Results: A total of 17 eligible studies were included in this pooled analysis, with 1777 cases and 1478 controls. The overall results were shown as follows: sensitivity, 0.74 (95\% $\mathrm{CI}=0.65-0.81)$, specificity, $0.79(95 \% \mathrm{CI}=0.73-0.84)$, positive likelihood ratio (PLR), 3.48 (95\% CI=2.79-4.32), negative likelihood, 0.33 (95\% CI=0.25-0.44), diagnostic score, 2.34 (95\% CI=1.99-2.69), diagnostic odds ratio, 10.41 (95\% CI=7.33-14.78) and area under the curve, $0.83(95 \% \mathrm{CI}=0.80-0.86)$. Deeks' funnel plot asymmetry test $(\mathrm{p}=0.66)$ suggested no potential publication bias.

Conclusion: All these results indicate that lncRNA MALAT1 achieves a relatively moderate accuracy in cancer detection and diagnosis, and could serve as a diagnostic biomarker for cancers.

Keywords: IncRNA, MALAT1, cancer, pooled analysis, detection, diagnosis

\section{Introduction}

With a leading cause of morbidity and mortality in recent years, cancer has become a significant public health problem that threatening peoples' life quality all over the world. ${ }^{1,2}$ Despite the rapid development of cancer treatments, such as surgery, chemotherapy, radiotherapy, and targeted therapy, the prognosis of patients with cancers still remains unsatisfactory. ${ }^{3-5}$ The lack of early diagnostic techniques and Delay in the diagnosis of cancer are the major obstacles in the current situation, contributing to missing the optimal opportunity for treatment, less chance of surviving, and more expensive costs. ${ }^{6-8}$ Thus, it is urgent to identify a novel diagnostic marker with good sensitivity and specificity for the early detection of cancer.

LncRNAs are often described as non-coding transcripts more than 200 nucleotides in length, lacking functional open reading frames (ORFs) and protein-coding capability. ${ }^{9,10}$ Previous studies have shown that lncRNAs perform multiple 
physiological and pathological biological functions. ${ }^{11}$ Aberrant expression of lncRNAs is widely observed in many cancers, involving in cancer initiation, progression, and metastasis. ${ }^{12-14}$ Furthermore, increasing evidence indicates that lncRNAs could serve as potential biomarkers in cancer detection and diagnosis, with high sensitivity and specificity. ${ }^{15,16}$

MALAT1, located at 11q13, was firstly characterized by $\mathrm{Ji}$ et $\mathrm{al}^{17}$ in a study of early-stage non-small-cell lung cancer, with a total $8.5 \mathrm{~kb}$ full length. A subsequent research study has demonstrated that MALAT1 is widely expressed in normal tissues, involving in cell viability and normal development. ${ }^{18}$ While MALAT1 was initially described as a prognostic marker of lung cancer metastasis, emerging evidence has shown that this IncRNA contributes greatly to other types of cancer's development and progression. ${ }^{19-22}$ Furthermore, an increasing number of studies suggest that the aberrant expression of MALAT1 in tumor tissues or body fluids may serve as a biomarker for tumor diagnosis and prognosis.

However, the diagnostic accuracy of MALAT1 in individual studies is still inconsistent and controversial. For instance, Liu et $\mathrm{al}^{23}$ revealed that MALAT1 achieve a moderate-high sensitivity and specificity of $86.0 \%$ and
$75.0 \%$ in the diagnosis of gastric cancer, respectively, whereas Yazarlou et $\mathrm{al}^{24}$ demonstrated that a relatively low sensitivity and specificity of $62.7 \%$ and $69.4 \%$, respectively, in bladder cancer detection. The agreement is hard to reach among these results because of ethnicity, study design, types of tumors, stage of cancer, and the small sample size. Previously, Mei et al performed a meta-analysis of MALAT1 expression in cancer detection for the first time, revealing that MALAT1 might be an effective cancer diagnostic marker. However, a limited number of cancer cases and small sample size in single tumor type make it unpersuasive enough. $^{25}$ Thus, we conducted this pooled analysis to summarize the overall diagnostic performance of MALAT1 in cancer detection and diagnosis and further explored its clinical value.

\section{Methods}

\section{Search Strategy and Study Eligibility Criteria}

Literature research was performed in the database including PubMed, Cochrane library, CNKI and Wanfang library, up to December 20, 2019, by the following searching strategy: "cancer" or "tumor" or "carcinoma" or "neoplasm" or "malignancy" or "neoplasm" and

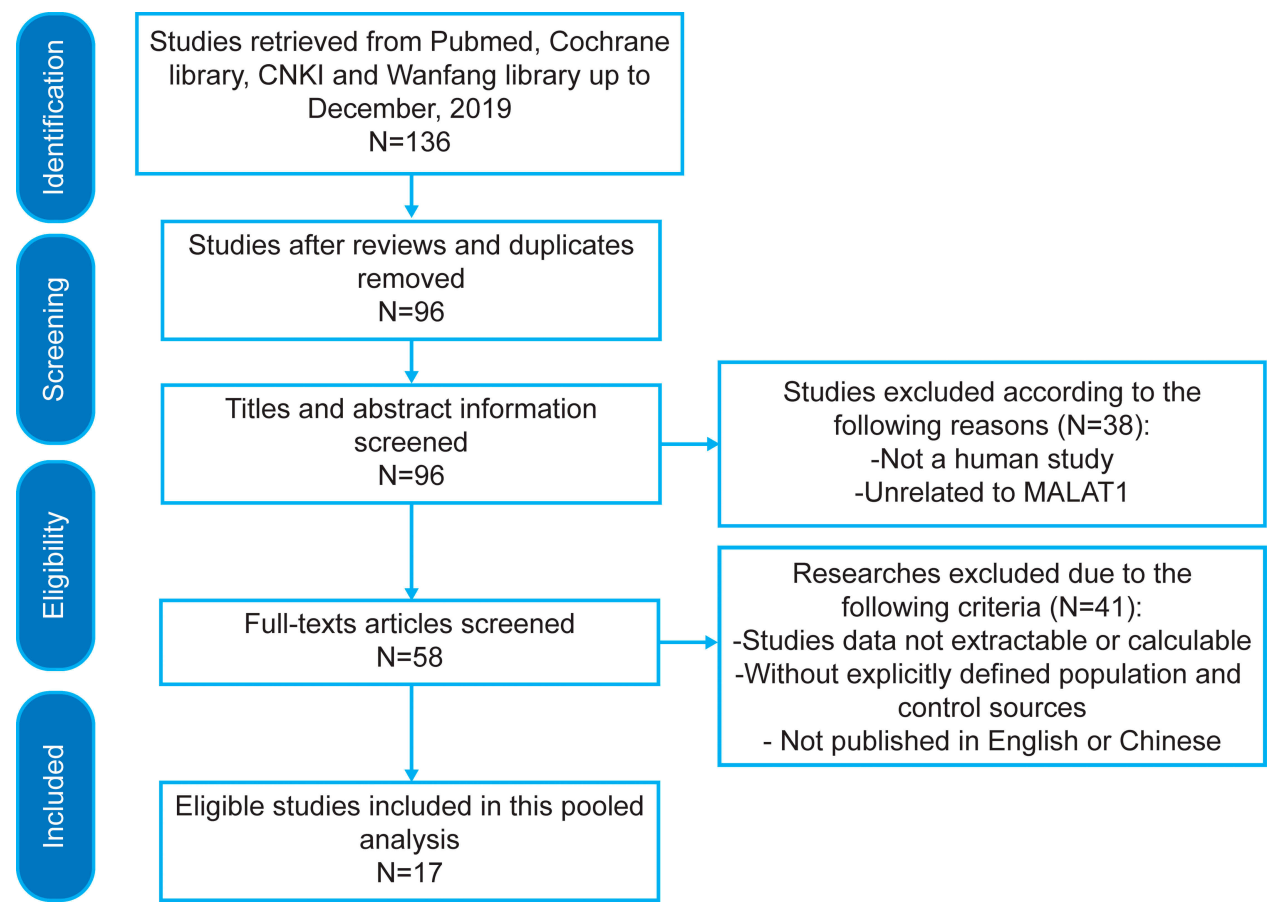

Figure I The flow diagram of relevant studies from the electronic databases.

Abbreviations: CNKI, China National Knowledge Infrastructure; MALATI, metastasis-associated lung adenocarcinoma transcript I. 


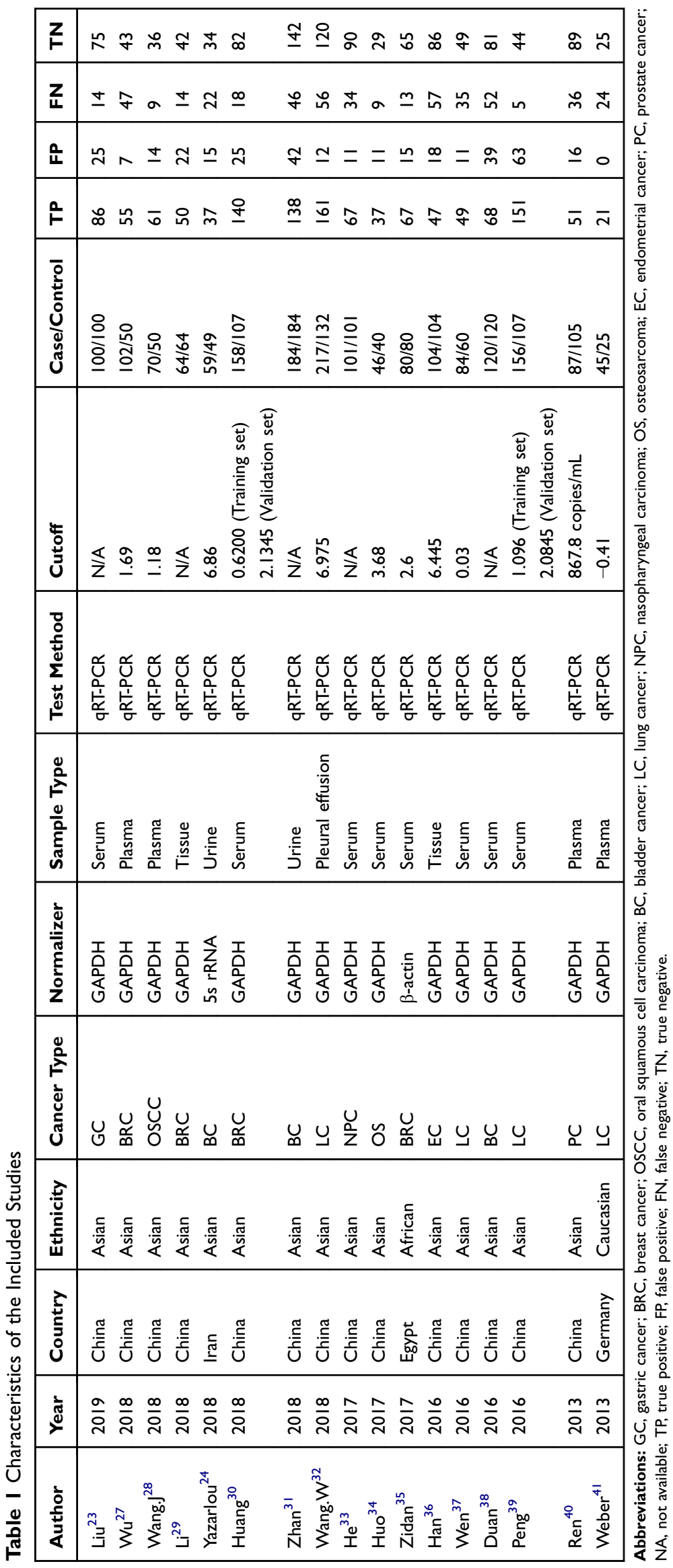


Table 2 Study Quality of the Diagnostic Studies Judged by the QUADAS II Checklist

\begin{tabular}{|c|c|c|c|c|c|c|c|c|c|}
\hline \multirow[t]{2}{*}{ Author } & \multirow[t]{2}{*}{ Year } & \multicolumn{4}{|c|}{ Risk of Bias } & \multicolumn{3}{|c|}{ Concerns Regarding Applicability } & \multirow{2}{*}{$\begin{array}{l}\text { Total } \\
\text { Stars }\end{array}$} \\
\hline & & $\begin{array}{l}\text { Patient } \\
\text { Selection }\end{array}$ & $\begin{array}{l}\text { Index } \\
\text { Test }\end{array}$ & $\begin{array}{l}\text { Reference } \\
\text { Standard }\end{array}$ & $\begin{array}{l}\text { Flow and } \\
\text { Timing }\end{array}$ & $\begin{array}{l}\text { Patient } \\
\text { Selection }\end{array}$ & $\begin{array}{l}\text { Index } \\
\text { Test }\end{array}$ & $\begin{array}{l}\text { Reference } \\
\text { Standard }\end{array}$ & \\
\hline $\mathrm{Liu}^{23}$ & 2019 & Low & Low & Low & Unclear & Low & Low & Low & 6 \\
\hline$W u^{27}$ & 2018 & Low & Low & Low & Unclear & Low & Low & Low & 6 \\
\hline Wang.j ${ }^{28}$ & 2018 & Low & Low & Low & Unclear & High & Low & Low & 5 \\
\hline $\mathrm{Li}^{29}$ & 2018 & Low & High & Low & Unclear & Unclear & Low & Low & 4 \\
\hline Yazarlou $^{24}$ & 2018 & Unclear & Unclear & Low & Low & Unclear & Low & Low & 4 \\
\hline Huang ${ }^{30}$ & 2018 & Low & Low & Low & Low & Unclear & Low & Low & 6 \\
\hline Zhan ${ }^{31}$ & 2018 & Low & Low & Low & Unclear & Unclear & Low & Low & 5 \\
\hline Wang. $W^{32}$ & 2018 & Low & Unclear & Low & Unclear & Low & Low & Low & 5 \\
\hline $\mathrm{He}^{33}$ & 2017 & Low & Unclear & Low & Low & Unclear & Low & Low & 5 \\
\hline $\mathrm{Huo}^{34}$ & 2017 & Unclear & Low & Low & Unclear & Unclear & Low & Low & 4 \\
\hline Zidan ${ }^{35}$ & 2017 & Unclear & Low & Low & Unclear & Unclear & Low & Low & 4 \\
\hline $\mathrm{Han}^{36}$ & 2016 & Low & High & Low & Unclear & Unclear & Low & Low & 4 \\
\hline $\mathrm{Wen}^{37}$ & 2016 & Low & Low & Low & Unclear & Low & Low & Low & 6 \\
\hline Duan $^{38}$ & 2016 & Low & Unclear & Low & Unclear & Unclear & Low & Low & 4 \\
\hline Peng ${ }^{39}$ & 2016 & Low & Low & Low & Low & High & Low & Low & 6 \\
\hline $\operatorname{Ren}^{40}$ & 2013 & Low & Low & Low & Low & Unclear & Low & Low & 6 \\
\hline Weber ${ }^{4 I}$ & 2013 & Unclear & Low & Low & Low & Low & Low & Low & 6 \\
\hline
\end{tabular}

"MALAT1" and "sensitivity" or "specificity" or "ROC curve" or "accuracy". Two investigators (YZ and YQY) check the titles and abstract information of the studies, and then scanned the full articles to delete irrelevant studies by using the following inclusion criteria: (1) human clinical studies; (2) the diagnostic value of lncRNA MALAT1 for detecting cancer evaluated in studies; (3) the number of true positive (TP), false positive (FP), false negative (FN) and true negative (TN) in cancer patients and controls could be drawn or calculated from the original included studies and (4) being published in English or Chinese. Accordingly, the articles were excluded on the basis of the following exclusion criteria: (1) laboratory studies on animal models or cell lines; (2) reviews, meta-analysis, case reports, commentaries and (3) lack of sufficient data to calculate TP, FP, $\mathrm{FN}$ and $\mathrm{TN}$.

\section{Data Extraction and Quality Assessment}

The data and primary information from each included study, including first author, year of publication, country, ethnicity, cancer type, Normalizer, sample type, test method, cutoff value, sample size, true positive (TP), false positive (FP), true negative (TN), and false negative (FN) were extracted by two reviewers ( $\mathrm{YZ}$ and YQY) independently and cross-checked. Divergences will come to an agreement by another two authors (SY and $\mathrm{CMZ}$ ).

\section{Quality Assessment}

The QUADAS-2 was applied to evaluate the quality of the studies included in this pooled analysis systematically. With the maximum QUADAS-2 score of 7, we can judge the quality of the included studies based on the score. Studies with four or more scores were defined as moderate-high quality. All of these were carried out independently by two authors (YZ and SY); Any disagreement was resolved in the conference by two authors (LW and WXZ).

\section{Statistical Analysis}

All statistical analysis were performed using Stata 14.0 (Stata Corporation, College Station, TX, USA). The pooled sensitivity, specificity, diagnostic score (DS), diagnostic odds ratio (DOR), positive likelihood ratio (PLR) and negative likelihood ratio (NLR) and other parameters were calculated by the bivariate model. Then, summary receiver operator characteristic (SROC) curves are applied to analyze and calculated the area under the ROC curves (AUC), to assess the overall diagnostic value of lncRNA MALAT1 in cancer detection and diagnosis. These data were confirmed by a hierarchical 


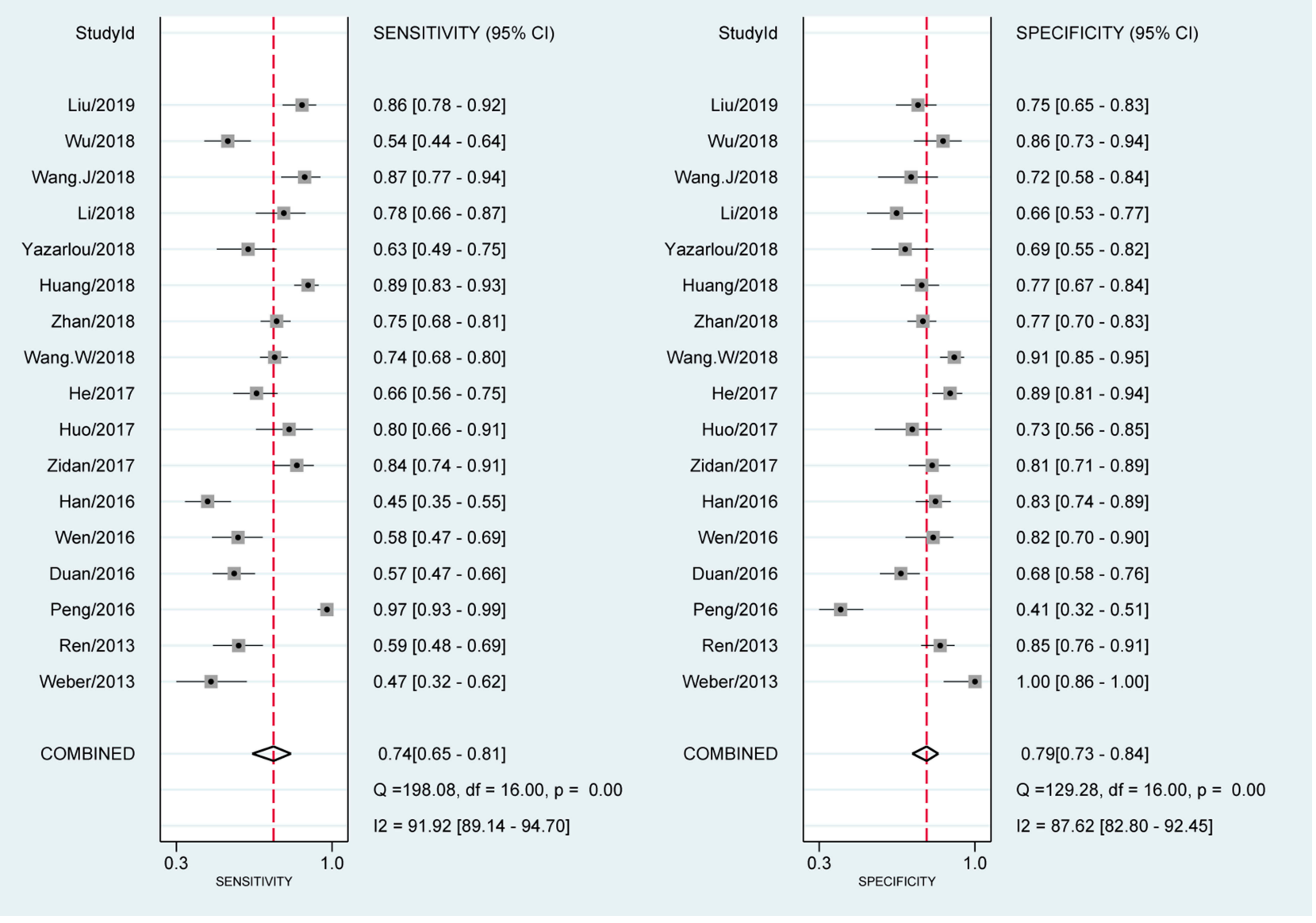

Figure 2 Forest plots of pooled sensitivity and specificity of 17 included studies.

summary receiver operating characteristics (HSROC) model. Cochran-Q and Inconsistency index $\left(\mathrm{I}^{2}\right)$ test were applied in order to evaluate the statistical heterogeneity across the included publications. A $P$ value less than 0.10 for the $\mathrm{Q}$ test or $\mathrm{I}^{2}$ value higher than $50 \%$ indicated obvious heterogeneity between the studies. ${ }^{26}$ In addition, Fagan's Nomogram was used to confirm relationships between prior-test probability, likelihood ratio, and posttest probability. Deeks' funnel plot was applied for publication bias evaluation.

\section{Results}

\section{Studies Selection and General Features of Included Studies}

A total of 17 eligible studies ${ }^{23,24,27-41}$ including 1777 cases and 1478 controls were finally included in the pooled analysis after a systematic search of PubMed, Cochrane library, CNKI and Wanfang library database from 2013 to 2019, according to inclusion and exclusion criteria (Figure 1). The main characteristics of the included studies were displayed in Table 1. In total, there were studies on breast cancer $(\mathrm{N}=4)$, lung cancer $(\mathrm{N}=4)$, bladder cancer $(\mathrm{N}=3)$, gastric cancer $(\mathrm{N}=1)$, oral squamous cell carcinoma $(\mathrm{N}=1)$, osteosarcoma $(\mathrm{N}=1)$, endometrial cancer $(\mathrm{N}=1)$, nasopharyngeal carcinoma $(\mathrm{N}=1)$ and prostate cancer $(\mathrm{N}=1)$. The expression of lncRNA MALAT1 was measured by qRT-PCR methods based on serum $(\mathrm{N}=8)$, plasma $(\mathrm{N}=4)$, tissue $(\mathrm{N}=2)$, urine $(\mathrm{N}=2)$ and Pleural effusion $(\mathrm{N}=1)$. In all included studies, the expression of MALAT1 showed an up-regulation trend in cancer samples when compared with controls.

\section{Quality Assessment}

The scores of the Quality Assessment of Diagnostic Accuracy Studies-2 (QUADAS-2) study quality assessment were shown in Table 2. Among the 17 studies, seven studies achieved 6 stars, four studies achieved 5 stars, and six studies achieved 4 stars, indicating a moderate-high quality for most of the studies. 


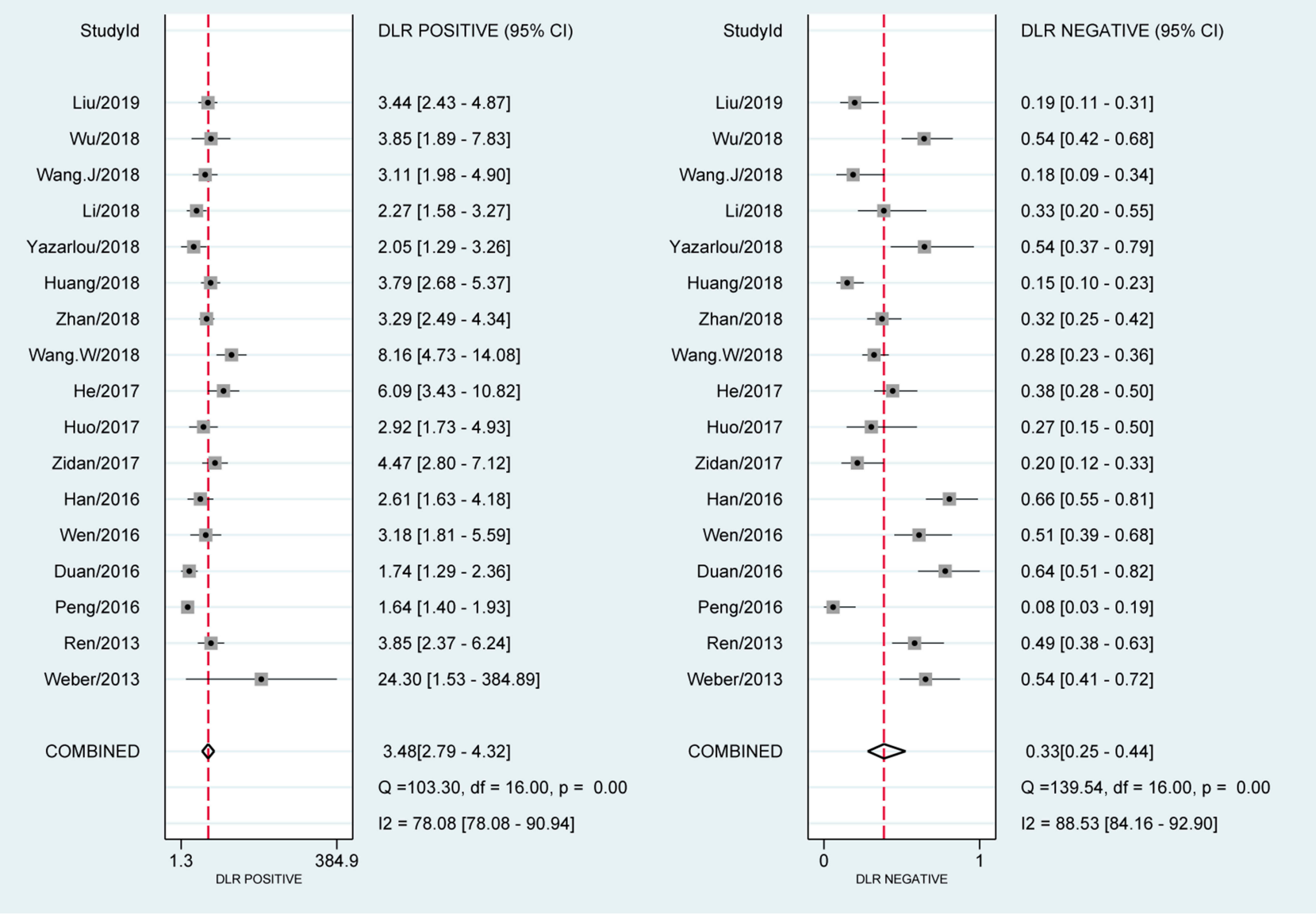

Figure 3 Forest plots of positive likelihood ratio (PLR) and negative likelihood ratio (NLR) for MALATI in the diagnosis of cancer. Abbreviation: DLR, diagnostic likelihood ratio.

\section{Pooled Results}

The forest plot of data from 17 included articles on sensitivity and specificity for MALAT1 in diagnosing cancer was shown in Figure 2. The sensitivity and specificity for the pooled data were 0.74 [ $95 \% \mathrm{CI}=0.65-0.81]$ and 0.79 [ $95 \% \mathrm{CI}=0.73-0.84]$, respectively. Significant heterogeneity was found for both sensitivity $\left[\mathrm{I}^{2}=91.92 \%, 95 \% \mathrm{CI}=89.14-94.70 \%\right]$ and specificity $\left[\mathrm{I}^{2}=87.62 \%, 95 \% \mathrm{CI}=82.80-92.45 \%\right]$. Meanwhile, we found that the pooled PLR was 3.48 [95\% CI $=2.79-4.32]$, the NLR was $0.33[95 \% \mathrm{CI}=0.25-0.44]$ (Figure 3). The DS and DOR were 2.34 [95\% CI=1.99-2.69] and 10.41 [95\% $\mathrm{CI}=7.33-14.78]$, respectively (Figure 4 ).

\section{Pooled SROC and HSROC}

The SROC curve for the 17 included studies was 0.83 [95\% $\mathrm{CI}=0.80-0.86]$, as shown in Figure 5, indicating a relatively moderate-high diagnostic value. Besides, The HSROC curve of these included studies was consistent with the results of the bivariate model. The calculated $\beta$ value was -0.29 (95\%
$\mathrm{CI}=-0.76-0.17$ ), and the $P$ value was 0.210 , implying that the HSROC was symmetrical (Figure 6).

\section{Clinical Utility of the Index Test}

We performed Fagan's Nomogram to predict the increasing inerrability about a positive diagnosis by using the value of the test, and it is used for estimating posttest probabilities. When MALAT1 assays were tested for all individuals with a pretest probability of $55 \%$ to have cancer, a positive result would improve posttest probability having cancer to $81 \%$, whereas a negative result would drop the posttest probability to $29 \%$ (Figure 7 ).

\section{Influence Analysis and Robustness Tests}

God-of-ft and bivariate normality analysis (Figure 8A and B) showed that the bivariate model was moderately robust. And then, we performed sensitivity analysis after further excluded 3 outliers found by influence analysis and outlier detection in Figure $8 \mathrm{C}$ and D. The sensitivity dropped 


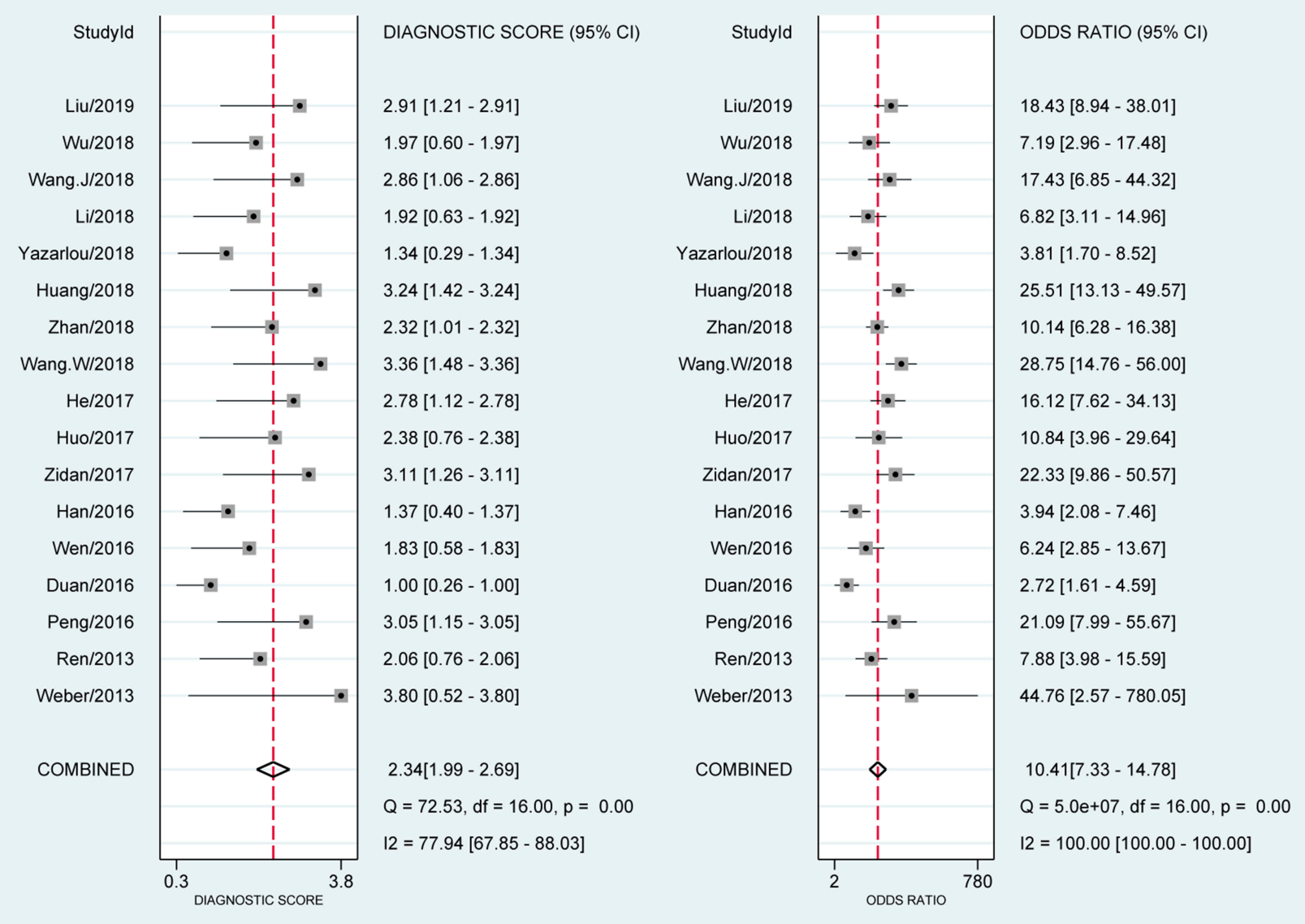

Figure 4 Forest plots of pooled diagnostic score (DS) and diagnostic odds ratio (DOR) for MALATI in the diagnosis of cancer.

from 0.74 to 0.73 , the specificity increased from 0.79 to 0.80 , the PLR increased from 3.48 to 3.69 , the NLR remain the same as 0.33 , the DS increased from 2.34 to 2.40 , the DOR increased from 10.41 to 11.04 , and the AUC increased from 0.83 to 0.84 , showing no significant change after the exclusion of the outliers (Supplementary Figures 1-3).

\section{Publication Bias Evaluation}

The publication bias was evaluated by Deeks' funnel plot in this pooled analysis and indicated no significant publication bias for MALAT1 $(P=0.66)$ (Figure 9).

\section{Subgroup Analysis}

According to the cancer type, we performed the subgroup analysis for lung cancer and breast cancer (Supplementary Table 1 and Supplementary Figure 4). The pooled sensitivity, specificity, PLR, DLR, DS, DOR and AUC for lung cancer were $0.76,0.85,5.02,0.28,2.88,17.73$ and 0.88 , respectively. Whereas for breast cancer, the pooled sensitivity, specificity, PLR, DLR, DS, DOR and AUC were $0.78,0.78,3.56,0.28,2.55,12.86$ and 0.84 , respectively. Meanwhile, subgroup analysis, according to different sample types, were also performed (Supplementary Table 2 and Supplementary Figure 5). The pooled results from studies based on serum sample detection were as follows: sensitivity, 0.81; specificity, 0.75; PLR, 3.18; NLR, 0.26; DS 2.52; DOR 12.43 and AUC, 0.84. As for studies based on plasma sample detection, the pooled results were as follows: sensitivity, 0.63; specificity, 0.85; PLR, 4.29; NLR, 0.43; DS 2.29; DOR 9.90 and AUC, 0.84.

\section{Discussion}

In recent years, IncRNA MALAT1 has been reported to participate in the prognosis of tumors by regulating a variety of biological processes, such as proliferation, apoptosis, invasion and angiogenesis. ${ }^{22,42-44}$ Furthermore, several studies demonstrated that aberrant expression of 


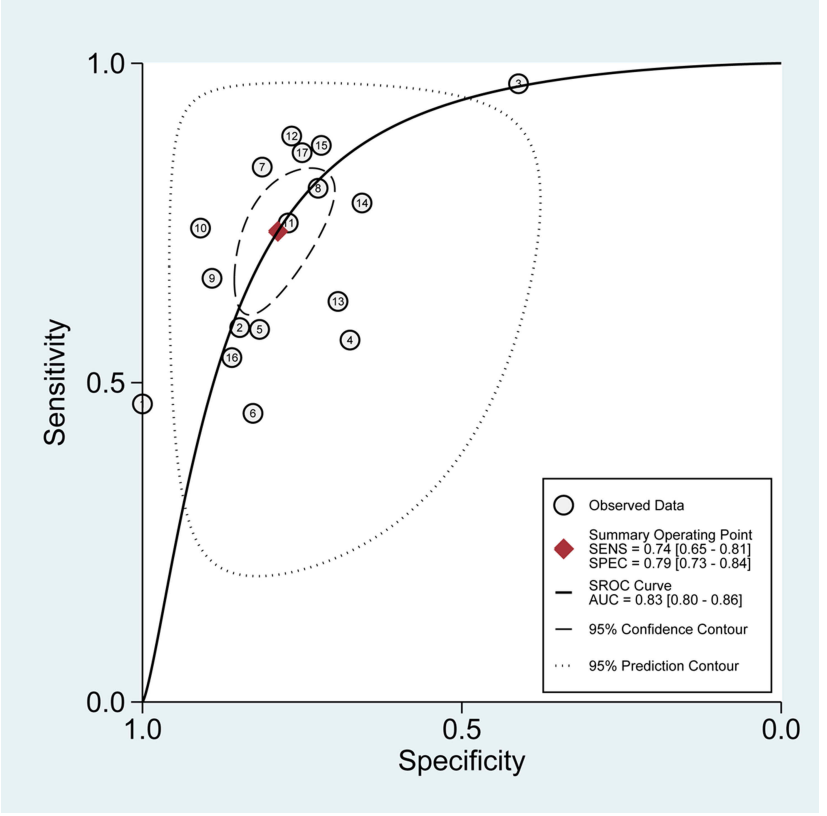

Figure 5 Summary receiver operating characteristic (SROC) graph of 17 included studies.

Abbreviations: SENS, sensitivity; SPEC, specificity; AUC, area under curve.

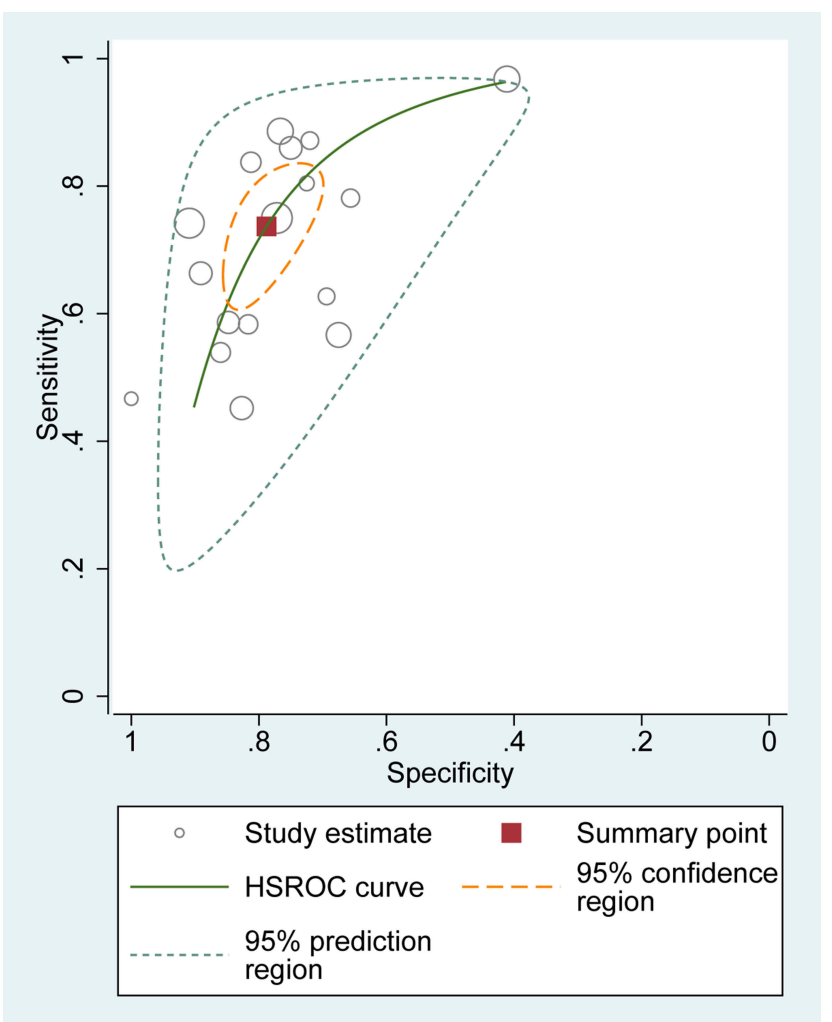

Figure 6 Hierarchical summary receiver operating characteristics (HSROC) curve for MALATI in the diagnosis of cancer.

MALAT1 in tumor tissues or body fluids might serve as a biomarker for tumor diagnosis and prognosis. ${ }^{28,29,31,32} \mathrm{An}$ ideal biomarker should be easily obtained with minimal risk

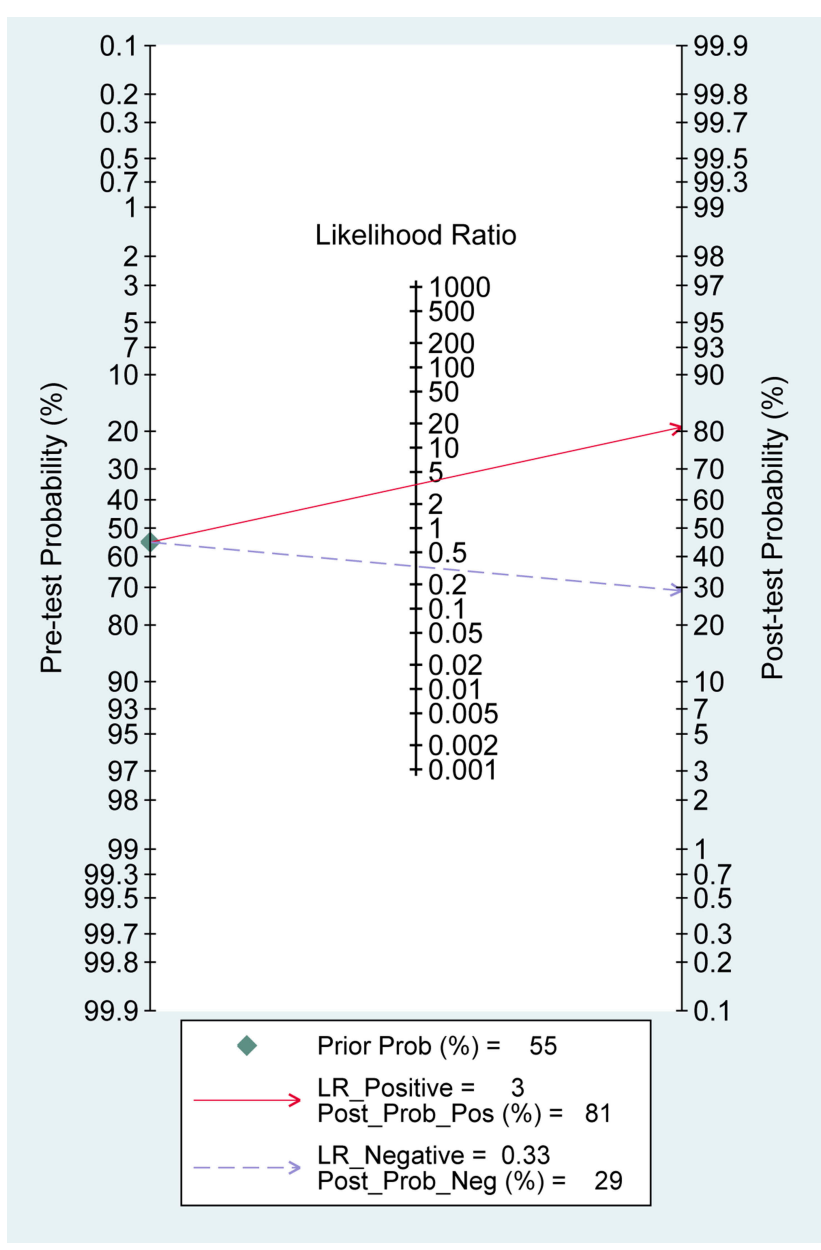

Figure 7 Fagan's Nomogram for calculation of posttest probabilities. Abbreviation: LR, likelihood ratio.

and discomfort to patients, as well as detectable and reproducible in standard clinical laboratories. Luckily, lncRNA MALAT1 possesses all these features as a biomarker. ${ }^{45}$ MALAT1 is not only expressed in tissues but also detectable in body fluids, such as blood and urine, ${ }^{24,38}$ which are easily obtainable with minimal damage to the patient. However, the diagnostic accuracy of MALAT1 still remains inconsistent and controversial. Thus, in our analysis, 17 studies with 3255 subjects were pooled to evaluate the diagnostic value of lncRNA MALAT1 in human cancers detection.

In the present pooled analysis, the results showed that the sensitivity, specificity and AUC of MALAT1 were 0.74, 0.79 and 0.83 , respectively, indicating a capacity to distinguish cancer patients from normal people. Meanwhile, a pooled PLR of 3.48 and NLR of 0.33 implied that patients with cancer have a 3.48-fold higher possibility of being MALAT1 positive for patients with cancer compared with controls, and $33 \%$ of all individuals have negative results, 
(a) Goodness-Of-Fit

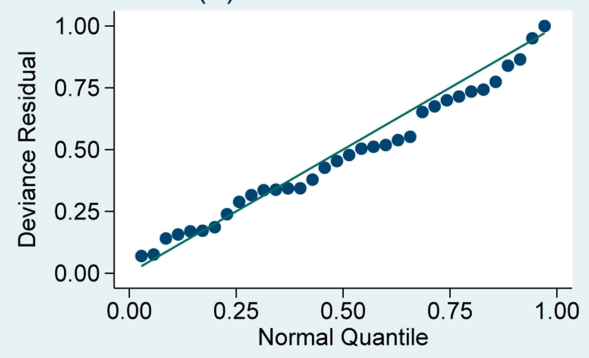

(c) Influence Analysis

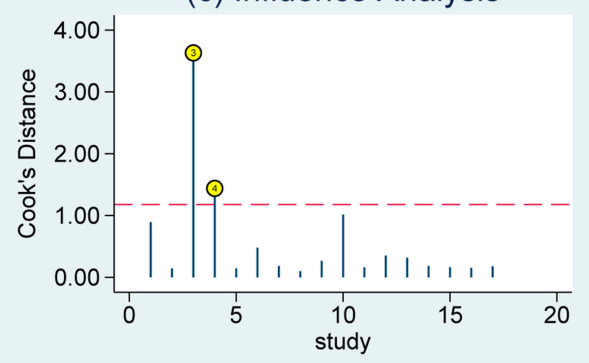

(b) Bivariate Normality

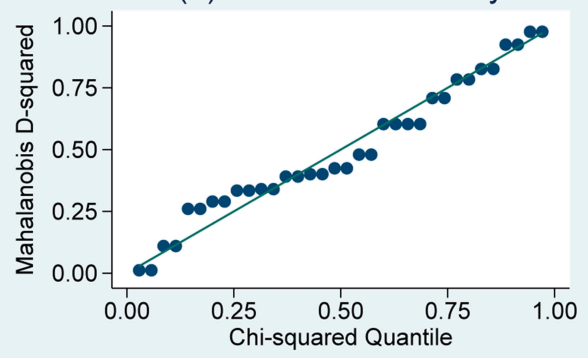

(d) Outlier Detection

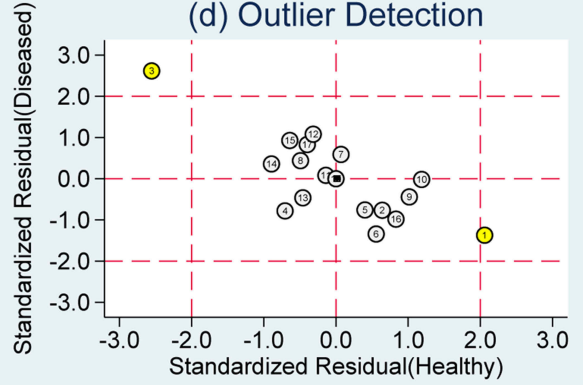

Figure 8 Graphs for sensitivity analysis: (A) goodness of fit, (B) bivariate normality, (C) influence analysis, and (D) outlier detection.

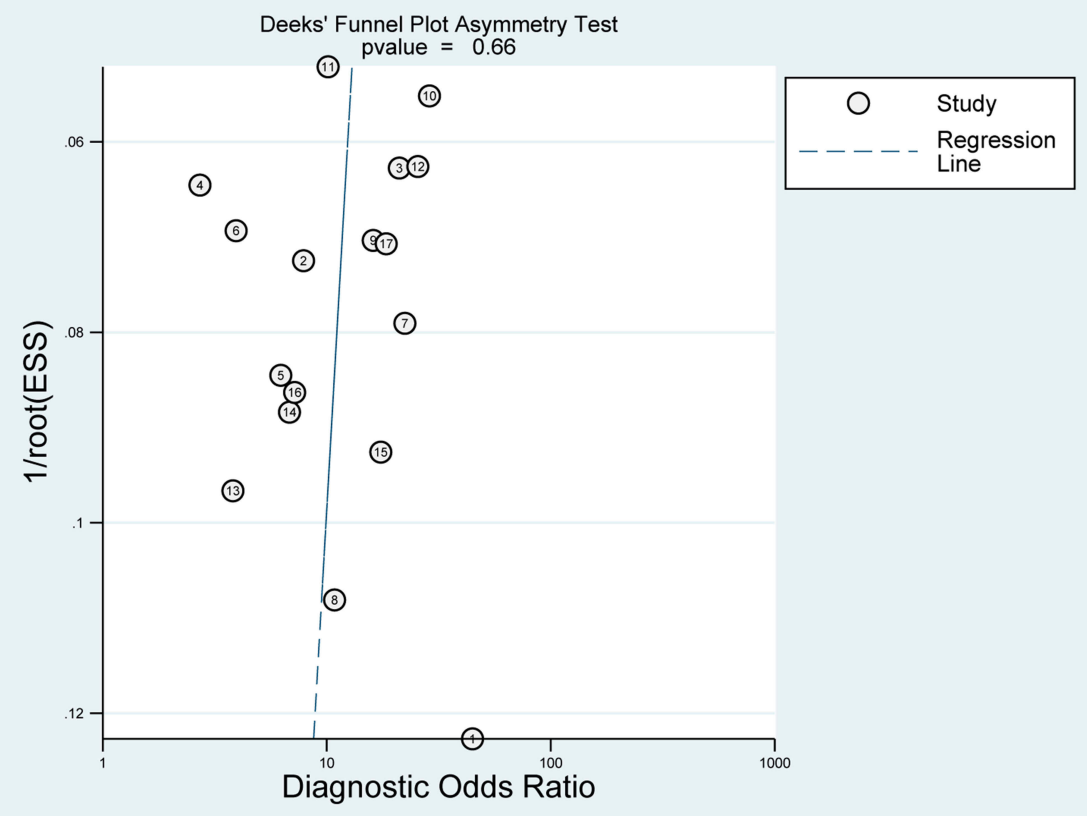

Figure 9 Graph of Deeks' funnel plot asymmetry test.

suggesting that the diagnostic value of MALAT1 is relatively moderate. Moreover, the pooled DS and DOR were 2.34 and 10.41, reflecting a moderate level of diagnostic accuracy. In addition, Fagan's nomogram showed MALAT1 could raise the probability of cancer detection by $26 \%$ (post-test probability $81 \%$, pre-test probability $55 \%$ ).

Similar analysis were also performed in lung cancer and breast cancer subgroup. The pooled AUC, DS and DOR 
were $0.88,2.88$ and 17.73 in lung cancer, and 0.84, 2.55 and 12.86 in breast cancer, indicating that MALAT1 could act as an effective diagnostic biomarker in both types of these two cancers. Meanwhile, MALAT1 achieved a high diagnostic value in circulating blood for cancer detection, with a pooled AUC of 0.84 , DS of 2.52 and DOR of 12.43 in serum sample detection, and an AUC of 0.84, DS of 2.29 and DOR of 9.90 in plasma sample detection. Furthermore, a pooled sensitivity of 0.81 and specificity of 0.75 in serum, and a pooled sensitivity of 0.63 and specificity of 0.85 in plasma, demonstrated that circulating MALAT1 had relatively moderate accuracy in human cancer detection.

The pooled analysis in our study also had its limitations. First, a very high ratio of data in Chinese populations was included in our analysis, which might contribute to inevitable publication bias. Second, not all of the studies reported the cutoff values of IncRNA MALAT1. Third, only studies published in English or Chinese were screened and included in the pooled analysis, studies published in other languages should not be ignored.

\section{Conclusion}

In summary, these pooled results demonstrated that IncRNA MALAT1 could serve as a detection and diagnosis biomarker in various human cancers, especially in serum/plasma, with a relatively moderate accuracy in distinguishing cancer patients from all individuals. We also proved that the moderate diagnostic value of MALAT1 in lung cancer and breast cancer subgroup; However, more cancer types studies with a large sample size are needed to strengthen our conclusion.

\section{Acknowledgments}

This work was supported by the National Natural Science Foundation of China (grant numbers 81871963, 81572335) and the China Scholarship Council (No:201906310088 to Yue Zhao).

\section{Disclosure}

All authors declare that there are no conflicts of interest.

\section{References}

1. Siegel RL, Miller KD, Jemal A. Cancer statistics, 2020. CA Cancer J Clin. 2020;70(1):7-30. doi:10.3322/caac.21590

2. Siegel RL, Miller KD, Jemal A. Cancer statistics, 2019. CA Cancer J Clin. 2019;69(1):7-34. doi:10.3322/caac.21551

3. Poon RT. Prevention of recurrence after resection of hepatocellular carcinoma: a daunting challenge. Hepatology. 2011;54(3):757-759. doi:10.1002/hep. 24569
4. Cree IA, Kurbacher CM, Lamont A, Hindley AC, Love S. A prospective randomized controlled trial of tumour chemosensitivity assay directed chemotherapy versus physicians? Choice in patients with recurrent platinum-resistant ovarian cancer. Anticancer Drugs. 2007;18(9):1093-1101. doi:10.1097/CAD.0b013e3281de727e

5. Yang K, Park W, Huh SJ, Bae DS, Kim BG, Lee JW. Clinical outcomes in patients treated with radiotherapy after surgery for cervical cancer. Radiat Oncol J. 2017;35(1):39-47. doi:10.3857/ roj.2016.01893

6. Car LT, Papachristou N, Urch C, et al. Preventing delayed diagnosis of cancer: clinicians' views on main problems and solutions. $J$ Glob Health. 2016;6(2):020901. doi:10.7189/jogh.06.020901

7. Gildea TR, DaCosta Byfield S, Hogarth DK, Wilson DS, Quinn CC. A retrospective analysis of delays in the diagnosis of lung cancer and associated costs. Clinicoecon Outcomes Res. 2017;9:261-269. doi:10.2147/CEOR.S132259

8. Bradley CJ. Cancer, Financial Burden, and Medicare Beneficiaries. J Clin Oncol. 2017;35(22):2461-2462. doi:10.1200/JCO.2017.73.1877

9. Bhan A, Soleimani M, Mandal SS. Long noncoding RNA and cancer: a new paradigm. Cancer Res. 2017;77(15):3965-3981. doi:10.1158/ 0008-5472.CAN-16-2634

10. Mercer TR, Mattick JS. Structure and function of long noncoding RNAs in epigenetic regulation. Nat Struct Mol Biol. 2013;20 (3):300-307. doi:10.1038/nsmb.2480

11. Beermann J, Piccoli MT, Viereck J, Thum T. Non-coding RNAs in development and disease: background, mechanisms, and therapeutic approaches. Physiol Rev. 2016;96(4):1297-1325. doi:10.1152/ physrev.00041.2015

12. Xie CR, Wang F, Zhang S, et al. Long noncoding RNA HCAL facilitates the growth and metastasis of hepatocellular carcinoma by acting as a ceRNA of LAPTM4B. Mol Ther Nucleic Acids. 2017;9:440-451. doi:10.1016/j.omtn.2017.10.018

13. Terashima M, Tange S, Ishimura A, Suzuki T. MEG3 long noncoding RNA contributes to the epigenetic regulation of epithelial-mesenchymal transition in lung cancer cell lines. J Biol Chem. 2017;292(1):82-99. doi:10.1074/jbc.M116.750950

14. Matsumura K, Kawasaki Y, Miyamoto $M$, et al. The novel G-quadruplex-containing long non-coding RNA GSEC antagonizes DHX36 and modulates colon cancer cell migration. Oncogene. 2017;36(9):1191-1199. doi:10.1038/onc.2016.282

15. Wang M, Dong X, Feng Y, Sun H, Shan N, Lu T. Prognostic role of the long non-coding RNA, SPRY4 intronic transcript 1 , in patients with cancer: a meta-analysis. Oncotarget. 2017;8(20):33713-33724. doi:10.18632/oncotarget.16735

16. Tian GW, Li N, Xin Y. Prognostic and clinicopathological significance of CCAT2 in Chinese patients with various tumors. Int J Biol Markers. 2017;32(3):e344-e351. doi:10.5301/ijbm.5000281

17. Ji P, Diederichs S, Wang W, et al. MALAT-1, a novel noncoding RNA, and thymosin beta4 predict metastasis and survival in early-stage non-small cell lung cancer. Oncogene. 2003;22 (39):8031-8041. doi:10.1038/sj.onc. 1206928

18. Eissmann M, Gutschner T, Hammerle M, et al. Loss of the abundant nuclear non-coding RNA MALAT1 is compatible with life and development. RNA Biol. 2012;9(8):1076-1087. doi:10.4161/rna.21089

19. Wang Z, Katsaros D, Biglia N, et al. High expression of long non-coding RNA MALAT1 in breast cancer is associated with poor relapse-free survival. Breast Cancer Res Treat. 2018;171(2):261-271. doi:10.1007/s10549-018-4839-2

20. Li C, Cui Y, Liu LF, et al. High expression of long noncoding RNA MALAT1 indicates a poor prognosis and promotes clinical progression and metastasis in bladder cancer. Clin Genitourin Cancer. 2017;15(5):570-576. doi:10.1016/j.clgc.2017.05.001

21. Malakar P, Shilo A, Mogilevsky A, et al. Long noncoding RNA MALAT1 promotes hepatocellular carcinoma development by SRSF1 upregulation and mTOR activation. Cancer Res. 2017;77 (5):1155-1167. doi:10.1158/0008-5472.CAN-16-1508 
22. Zhuang M, Zhao S, Jiang Z, et al. MALAT1 sponges miR-106b-5p to promote the invasion and metastasis of colorectal cancer via SLAIN2 enhanced microtubules mobility. EBioMedicine. 2019;41:286-298. doi:10.1016/j.ebiom.2018.12.049

23. Liu Y, Jiang M, Ni L, Xu Z, Xu J. Diagnostic value of serum carcinoembryonic antigen and carbohydrate antigen 724 combined with long non-coding RNA MALAT1 detection in gastric cancer Cancer Res Clin. 2019;31:88-92.

24. Yazarlou F, Modarressi MH, Mowla SJ, et al. Urinary exosomal expression of long non-coding RNAs as diagnostic marker in bladder cancer. Cancer Manag Res. 2018;10:6357-6365. doi:10.2147/ CMAR.S186108

25. Mei H, Liu Y, Zhou Q, Hu K, Liu Y. Long noncoding RNA MALAT1 acts as a potential biomarker in cancer diagnosis and detection: a meta-analysis. Biomark Med. 2019;13(1):45-54. doi:10.2217/bmm2018-0128

26. Rutter CM, Gatsonis CA. A hierarchical regression approach to meta-analysis of diagnostic test accuracy evaluations. Stat Med. 2001;20(19):2865-2884. doi:10.1002/sim.942

27. Wu J, Yu L, Wang X, Li J, Zhu M, Yan F. Expression and clinical significance of plasma MALAT1 in patients with breast cancer. Chin $J$ Lab Med. 2018;41:92-96.

28. Wang J, Zhao S, Ouyang S, Liao L. Expression and clinical significance of plasma long non-coding RNA MALAT1 in patients with oral squamous cell carcinoma. J Pract Stomatol. 2018;34:188-192.

29. Li Z, Zhou J, Zhou L, Yang H, Li M. Expression and clinical significance of long non-coding RNA MALAT1 in breast cancer $J$ Med Res. 2018;47:79-81.

30. Huang SK, Luo Q, Peng H, et al. A panel of serum noncoding RNAs for the diagnosis and monitoring of response to therapy in patients with breast cancer. Med Sci Monit. 2018;24:2476-2488. doi:10.12 659/MSM.909453

31. Zhan Y, Du L, Wang L, et al. Expression signatures of exosomal long non-coding RNAs in urine serve as novel non-invasive biomarkers for diagnosis and recurrence prediction of bladder cancer. Mol Cancer. 2018;17(1):142. doi:10.1186/s12943-018-0893-y

32. Wang WW, Zhou XL, Song YJ, Yu CH, Zhu WG, Tong YS. Combination of long noncoding RNA MALAT1 and carcinoembryonic antigen for the diagnosis of malignant pleural effusion caused by lung cancer. Onco Targets Ther. 2018;11:2333-2344. doi:10.2147/ OTT.S157551

33. He B, Zeng J, Chao W, et al. Serum long non-coding RNAs MALAT1, AFAP1-AS1 and AL359062 as diagnostic and prognostic biomarkers for nasopharyngeal carcinoma. Oncotarget. 2017;8 (25):41166-41177. doi:10.18632/oncotarget.17083
34. Huo Y, Li Q, Wang X, et al. MALAT1 predicts poor survival in osteosarcoma patients and promotes cell metastasis through associating with EZH2. Oncotarget. 2017;8(29):46993-47006. doi:10.18632/ oncotarget. 16551

35. Zidan HE, Karam RA, El-Seifi OS, Abd Elrahman TM. Circulating long non-coding RNA MALAT1 expression as molecular biomarker in Egyptian patients with breast cancer. Cancer Genet. 2018;220: 32-37. doi:10.1016/j.cancergen.2017.11.005

36. Han C. The expression and clinical significance of long non-coding RNA MALAT1 in endometrial cancer. Chin J Family Plann Gynecotokology. 2016;8:51-55.

37. Wen $\mathrm{Z}$, Li X. Diagnostic value of long non-coding RNA metastasis-associated lung adenocarcinoma transcript 1 in serum for clinical diagnosis of lung cancer. J Clin Pulm Med. 2016;21: 2154-2158.

38. Duan W, Du L, Jiang X, et al. Identification of a serum circulating lncRNA panel for the diagnosis and recurrence prediction of bladder cancer. Oncotarget. 2016;7(48):78850-78858. doi:10.18632/oncotarget. 12880

39. Peng H, Wang J, Li J, et al. A circulating non-coding RNA panel as an early detection predictor of non-small cell lung cancer. Life Sci. 2016;151:235-242. doi:10.1016/j.lfs.2016.03.002

40. Ren S, Wang F, Shen J, et al. Long non-coding RNA metastasis associated in lung adenocarcinoma transcript 1 derived miniRNA as a novel plasma-based biomarker for diagnosing prostate cancer. Eur J Cancer. 2013;49(13):2949-2959. doi:10.1016/j.ejca.2013.04.026

41. Weber DG, Johnen G, Casjens S, et al. Evaluation of long noncoding RNA MALAT1 as a candidate blood-based biomarker for the diagnosis of non-small cell lung cancer. BMC Res Notes. 2013;6(1):518. doi:10.1186/1756-0500-6-518

42. Liao K, Lin Y, Gao W, et al. Blocking lncRNA MALAT1/miR-199a/ ZHX1 axis inhibits glioblastoma proliferation and progression. Mol Ther Nucleic Acids. 2019;18:388-399. doi:10.1016/j.omtn.2019.09. 005

43. Hu Y, Lin J, Fang $\mathrm{H}$, et al. Targeting the MALAT1/PARP1/LIG3 complex induces DNA damage and apoptosis in multiple myeloma. Leukemia. 2018;32(10):2250-2262. doi:10.1038/s41375-018-0104-2

44. Qiu JJ, Lin XJ, Tang XY, Zheng TT, Lin YY, Hua KQ. Exosomal metastasis associated lung adenocarcinoma transcript 1 promotes angiogenesis and predicts poor prognosis in epithelial ovarian cancer. Int J Biol Sci. 2018;14(14):1960-1973. doi:10.7150/ijbs.28 048

45. Li ZX, Zhu QN, Zhang HB, Hu Y, Wang G, Zhu YS. MALAT1: a potential biomarker in cancer. Cancer Manag Res. 2018;10: 6757-6768. doi:10.2147/CMAR.S169406
OncoTargets and Therapy

\section{Publish your work in this journal}

OncoTargets and Therapy is an international, peer-reviewed, open access journal focusing on the pathological basis of all cancers, potential targets for therapy and treatment protocols employed to improve the management of cancer patients. The journal also focuses on the impact of management programs and new therapeutic

Submit your manuscript here: https://www.dovepress.com/oncotargets-and-therapy-journa agents and protocols on patient perspectives such as quality of life, adherence and satisfaction. The manuscript management system is completely online and includes a very quick and fair peer-review system, which is all easy to use. Visit http://www.dovepress.com/ testimonials.php to read real quotes from published authors. 